\title{
Dengue
}

\section{EPIDEMIOLOGÍA Y VIGILANCIA}

\section{Programa de control del dengue en Cali, 1998-2010, logros y retos}

Carlos Andrés Morales, María Eugenia Cuadros, Elizabeth Orobio, Harvey Vargas

Secretaría de Salud Pública, Cali, Colombia

Introducción. La situación del dengue en las Américas se ha agravado durante los últimos años; en el 2009 se reportaron 1'135.663 casos de dengue, 34.622 casos de dengue grave y 618 muertes -la cifra más alta en 30 años-, y en el 2010 se registró la cifra más alta de casos en toda la historia, 1'876.589.

Desde 1998 se reformuló el programa de control del dengue, enmarcado en la estrategia del control integrado y selectivo, con un manejo operativo con decisiones dependiente de los resultados de las continuas evaluaciones.

Materiales y métodos. Se priorizó el control intensivo de larvas de Aedes aegypti en los criaderos más productivos, 52.000 sumideros de aguas lluvia, y en los sitios de mayor riesgo de transmisión, 4.792 concentraciones humanas, mediante la implementación de la participación comunitaria, además de la vigilancia epidemiológica para el control de casos nuevos, la capacitación del personal operativo y la investigación operativa.

Resultados. En el periodo de 1989 a 1999 se registraron en Cali 33.258 casos de dengue, que al compararlos con 30.126 casos entre 2000 y 2010, muestra una reducción de 9,4\%, mientras que en Colombia y en las Américas el dengue aumentó en este último periodo en 118 y $208 \%$, respectivamente.

El índice de larvas en sumideros de aguas Iluvia disminuyo de $17,9 \%$ en 1998 a un promedio de $4,9 \%$ de 1999 a 2010. En las concentraciones humanas se redujeron los índices de predio y de Breteau en $49,1 \%$ y 63,4 \%, respectivamente.

Se ha logrado que, en promedio, el 52,1\% de las concentraciones humanas de la ciudad implemente medidas de control en las mismas: 19,0 \% aplican larvicidas, 26,1 \% instalaron mallas y 54,8 \% utilizaron otras medidas de control (secado, modificación de diseño, lavado y otras).

Conclusiones. Se han logrado importantes resultados en la disminución de los casos de dengue y de los índices entomológicos; este programa se puede tomar como modelo para ser implementado en otras ciudades.

\section{$\bullet \bullet$ \\ Influencia del evento climático El Niño sobre la dinámica de transmisión de dengue en Medellín, Colombia}

Guillermo Rúa-Uribe' ${ }^{1}$, Carolina Suárez-Acosta¹, Raúl

Rojo $^{2}$, Enrique Henao ${ }^{2}$, Rita Almanza ${ }^{3}$

1 Grupo Entomología Médica, Facultad de Medicina, Universidad de Antioquia, Medellín, Colombia

2 Programa de Inspección, Vigilancia y Control de Vectores y Zoonosis, Secretaría de Salud, Medellín, Colombia

3 Programa de Epidemiología Secretaría de Salud, Medellín, Colombia

Introducción. El dengue es una importante enfermedad de salud pública afectada por diferentes factores. Entre éstos, se ha evidenciado que la variabilidad climática influye en la incidencia de la enfermedad al afectar la dinámica de la población de los vectores y el período de desarrollo intrínseco del virus. La variabilidad climática local es afectada, a su vez, por elementos macroclimáticos, como el fenómeno de El Niño.

En el presente estudio se evaluó la relación entre los indicadores oceánicos del El Niño y la transmisión de dengue en Medellín con el propósito de generar información para la construcción de modelos estadísticos de predicción.

Materiales y métodos. Para el análisis de la información se emplearon las escalas temporales de mes, semana y período epidemiológico. El período de análisis comprendió desde la primera semana de 2001 hasta la semana 52 de 2010 (520 semanas epidemiológicas). Se hicieron análisis de autocorrelación, tendencia, variación estacional y regresión.

Resultados. Se observó que en los años posteriores a la ocurrencia del evento El Niño se incrementó la incidencia de la enfermedad en la ciudad. Mediante un análisis cruzado de relación se estimó que la incidencia de la enfermedad en la ciudad se asoció significativamente con la temperatura superficial del mar de la región El Niño 3,4 , con un rezago de 3 a 6 meses.

Conclusiones. La incidencia de dengue en Medellín se relaciona con la fluctuación de los 
indicadores macroclimáticos del evento El Niño. Esta información se convierte en una herramienta epidemiológica importante, la cual podrá ser incorporada a los sistemas de vigilancia de dengue de la ciudad para ayudar a mitigar el impacto de futuras epidemias de la enfermedad.

$$
\text { - } \bullet
$$

\section{Saberes y prácticas del dengue en El Carmen de Bolívar, 2010}

Martha Hernández

Secretaría Departamental de Salud de Bolívar, Cartagena, Colombia

Introducción. Se necesita comprender la atención en salud relacionada con el dengue a partir de los saberes y prácticas preventivas que tienen las personas responsables del almacenamiento y los cuidados de los depósitos de agua. Esto se puede lograr a partir de la identificación de los patrones sociales clave relacionados con los saberes y la prácticas preventivas de las personas responsables del almacenamiento del agua y cuidados de los depósitos, en el marco contexto sociocultural de los participantes; la exploración de las motivaciones y oportunidades para el mejoramiento de las prácticas preventivas, desde la participación comunitaria, que pudiera ser utilizada como evaluación de las actividades hasta ahora implementadas, lo que permitirá orientar el mejoramiento de las mismas y su aplicación en contextos similares.

Metodología. Se trata de una investigación cualitativa de tipo etnográfica, fundamentada en tres grandes periodos: definición de la situación problema, diseño y preparación del trabajo de campo e identificación de los patrones culturales; se utilizó la observación de los participantes y entrevistas semiestructuradas con una guía; la información fue suministrada por 18 informantes clave.

Resultados. Se evidenció ausencia de servicios de acueducto, alcantarillado, recolección y eliminación de residuos sólidos, bajo nivel educativo, y actividades de reacción en la presentación de casos. Se clasificaron e identificaron las manifestaciones de la enfermedad, los cuidados brindados por los familiares, la multicausalidad de la enfermedad, se reconocieron y se practicaron algunas medidas preventivas. Se develaron los patrones culturales de acudir al médico cuando el dengue era grave, nadie hacía lo que tenía que hacer y, a pesar de todo, algo se hizo.

Conclusiones. El incremento de la enfermedad está ligado a condiciones socioculturales, económicas y políticas, y al deficiente saneamiento básico; al concepto de acudir al médico cuando el dengue es malo, a la irregularidad de las acciones por parte de las instituciones y de la comunidad; no hay un abordaje integral del problema.

\section{Conocimientos, actitudes y prácticas con relación al dengue en la población adulta que acude diariamente al centro histórico de Cartagena}

Olida Castilla, Marianela Severiche, Alexi Ramos, Dilia Aparicio

Programa de Medicina, Corporación Universitaria Rafael Núñez, Cartagena, Colombia

Introducción. En la actualidad, la probabilidad de contraer dengue supera los 2.000 millones de personas, siendo la décima causa de muerte por enfermedades infecciosas. En Cartagena, la incidencia aumentó significativamente en los últimos tres años, considerándose una ciudad endémica dados los factores que predisponen a ella.

Objetivo. El objetivo fue identificar los conocimientos, actitudes y prácticas para la prevalencia y la incidencia con relación al dengue.

Materiales y métodos. Se utilizó un estudio descriptivo de corte de Cartagena durante el periodo de agosto a noviembre de 2010; se calculó una muestra de 150 personas tomadas al azar de las que acuden diariamente al centro histórico de la ciudad, y se les encuestó.

Resultados. Se encontró que de los encuestados, el $53,7 \%$ era de sexo masculino y $46,3 \%$ del femenino. El nivel de escolaridad más frecuente fue el técnico con $42,3 \%$. En lo que concierne al mecanismo de trasmisión, $72 \%$ manifestó que era por la picadura de un mosquito y $36,8 \%$ manifestó que el dengue se trasmitía de persona a persona. En el ítem que correspondía a la responsabilidad en la prevención del dengue, 38,5\% respondió que era del DADIS y $22 \%$ que de la comunidad; por último, se evaluó lo que se podría hacer para prevenir el dengue, la mayoría manifestó que la fumigación $(60 \%)$ y ante la pregunta de que era el dengue, $69,3 \%$ respondió que era una enfermedad trasmitida por un mosquito.

Conclusión. Este estudio abre las posibilidades del reconocimiento de la información que tienen los ciudadanos sobre el dengue y de la manera como se puede ayudar a detener la propagación del vector transmisor. 
Comandos Estudiantiles Antidengue: una novedosa estrategia de participación y movilización comunitaria en Medellín

Raúl Rojo', Enrique Henao', Rita Almanza', Ana Milena Zea $^{2}$, Guillermo Rúa-Uribe ${ }^{2}$

1 Secretaría de Salud, Medellín, Colombia

2 Grupo Entomología Médica, Universidad de Antioquia, Medellín, Colombia

Introducción. El dengue es una enfermedad multifactorial en la que los aspectos climáticos, entomológicos, económicos y culturales juegan un papel fundamental. La participación comunitaria es primordial en la implementación de los programas integrales de prevención y control.

Una estrategia desarrollada en Medellín y enfocada a la movilización comunitaria fue la conformación de los Comandos Estudiantiles Antidengue, un grupo de 15 estudiantes, aproximadamente, por institución educativa, encargados de la realización de actividades de promoción y prevención del dengue en su entorno escolar y familiar. El propósito fundamental en la conformación de los Comandos Estudiantiles Antidengue ha sido el generar un cambio de conducta en un grupo de la población en donde dicho cambio sea asimilable y permanente.

Materiales y métodos. Desde el 2008 en Medellín se vienen realizando actividades de participación y movilización comunitaria en las instituciones educativas de la ciudad. Estas actividades han incluido talleres de capacitación terórico-práctica, diseño y evaluación en las aulas de clase de pruebas biológicas de control vectorial y creación de material educativo, entre otras actividades.

Resultados. Se han conformado en la ciudad más de 200 Comandos Estudiantiles Antidengue, lo cual equivale a disponer de 3.000 personas, aproximadamente, participando en la implementación de las estrategias de promoción y prevención. Desde el 2007 se han capacitado 751.363 estudiantes y 21.436 profesores. Los Comandos EstudiantilesAntidenguehancontribuido al control social del dengue, fundamentalmente en la búsqueda y eliminación de criaderos.

Conclusión. Esta estrategia de participación y movilización comunitaria ha permitido involucrar a la población escolar en los programas de control del dengue realizados en la ciudad, generando un mayor conocimiento de la enfermedad y cambios de conducta. Se requiere continuar con este tipo de estrategias para vincular una mayor población escolar y consolidar el cambio de conducta hacia hábitos que promuevan la salud y prevengan el dengue en la ciudad. 